\title{
Estimating the costs of rearing young dairy cattle in the Netherlands using a simulation model that accounts for uncertainty related to diseases
}

\begin{abstract}
The costs of rearing young dairy cattle are a part of the cost of the price of milk, as rearing produces the future dairy cows. As most dairy farmers are not aware of the rearing costs, the rearing of dairy replacements often does not get the attention it deserves. Calculating the distribution of the rearing costs throughout the rearing process is difficult as the costs are correlated with biological processes, such as variation in growth rate and disease uncertainty. In this study, a calf level simulation model was built to estimate the rearing costs and their distribution from 2 weeks of age until first calving in the Netherlands. The uncertainties related to calf diseases (calf scours and bovine respiratory disease) were included, in which both the probabilities of disease and the effects of diseases (growth reduction) differ at different ages. In addition, growth was modeled stochastically and in a detailed manner using a two-phase growth function. The total cost of rearing young dairy cattle was estimated as $€ 1567$ per successfully reared heifer and varied between $€ 1423$ and $€ 1715$. Reducing the age of first calving by 1 month reduced the total cost between $2.6 \%$ and $5.7 \%$. The difference in the average cost of rearing between heifers that calved at 24 months and those calving at 30 months was $€ 400$ per heifer reared. Average rearing costs were especially influenced by labor efficiency and cost of feed. The rearing costs of a heifer that experienced disease at least once (20\% of the simulated heifers) were on average $€ 95$ higher than those of healthy heifers. Hence, for an individual diseased heifer, disease costs can be rather high, while the relative contribution to the average rearing cost for a standard Dutch dairy farm is low (approx. 3\%). Overall, the model developed proved to be a useful tool to investigate the total cost of rearing young dairy cattle, providing insights to dairy farmers with respect to the cost-efficiency of their own rearing management.
\end{abstract}

Keyword: Young dairy cattle rearing; Simulation model; Calf diseases; Economics 